\title{
Perforated Sigmoid Colon Cancer within an Irreducible Inguinal Hernia: a Case Report
}

Kai-Hsiung Ko, MD'

Chih-Yung Yu, MD ${ }^{1}$

Chien-Chang Kao, MD²

Shih-Hung Tsai, MD ${ }^{3}$

Guo-Shu Huang, MD'

Wei-Chou Chang, MD ${ }^{1}$

\section{Index terms :}

Perforation

Sigmoid adenocarcinoma

Inguinal hernia

Computed tomography (CT)

DOI:10.3348/kjr.2010.11.2.231

Korean J Radiol 2010; $11: 231-233$

Received June 3, 2009; accepted

after revision August 28, 2009.

'Department of Radiology, ${ }^{2}$ Division of Genitourinary Surgery, Department of Surgery and ${ }^{3}$ Department of Emergency Medicine, Tri-Service General Hospital,

National Defense Medical Center, China

Address reprint requests to:

Wei-Chou Chang, MD, Department of Radiology, Tri-Service General Hospital,

325, Cheng-Kung Road, Sec. 2, Taipei,

114, Taiwan, Republic of China.

Tel. (8862) 8792-7244

Fax. (8862) 8792-7245

e-mail: chougo2002@yahoo.com.tw
A perforated sigmoid colon cancer within an inguinal hernia is extremely rare. This unexpected finding is usually discovered during surgery and causes an unavoidable septic evolution. Here, we describe the case of an 84-year-old man who presented with fever, abdominal distension, and a painful, enlarged, left scrotum. A CT showed a left, incarcerated, inguinal hernia containing a perforated sigmoid adenocarcinoma (which was confirmed by histopathology). The possibility of an irreducible inguinal hernia in association with perforated sigmoid colon cancer should be considered in the array of diagnoses. A pre-operative CT scan would be helpful in facilitating an accurate diagnosis.

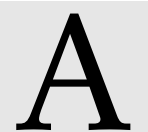

$\mathrm{n}$ inguinal hernia is usually reducible and without symptoms. However, in about $10 \%$ of cases, incarceration occurs, which may be due to benign or malignant lesions (1). The perforation of a sigmoid colon cancer presenting as an incarcerated inguinal hernia is an extremely rare condition. A review of the literature indicated that there have been only three cases reported, and this critical condition was only found during surgery. Unavoidable intra-abdominal sepsis due to perforated colon cancer with surrounding abscesses in the scrotal sac often leads to fatal complications. Herein, we report such a rare case and emphasize the value of computed tomography $(\mathrm{CT})$ for the pre-operative diagnosis.

\section{CASE REPORT}

An 84-year-old man was admitted to our emergency department complaining of pain and swelling of the left scrotum associated with fever, chills, and abdominal distension. These symptoms had been present for one week. The patient's medical history revealed a 20 -year-old unrepaired left inguinal hernia and type II diabetes mellitus that was poorly controlled.

Upon physical examination, the patient had decreased blood pressure (89/66 $\mathrm{mmHg})$, an elevated temperature $\left(38.5^{\circ} \mathrm{C}\right)$ and tachycardia (110 beats $\left./ \mathrm{min}\right)$. An abdominal examination showed distension and decreased bowel sounds. The left inguino-scrotal region presented as being tender, erythematosus, and with an irreducible mass. The presumed diagnosis was an incarcerated hernia. The results of the laboratory evaluations were as follows: hemoglobin, $10.1 \mathrm{~g} / \mathrm{dL}$ (normal: 13.5-18 $\mathrm{g} / \mathrm{dL}$ ); white blood cell count, 16,300/ $\mu \mathrm{L}$ (normal: 4,500-11,000/ $\mu \mathrm{L}$ ); glucose, 541 $\mathrm{mg} / \mathrm{dL}$ (normal: 70-105 mg/dL) and C-reactive protein, $14.8 \mathrm{mg} / \mathrm{dL}$ (normal: $<0.5$ $\mathrm{mg} / \mathrm{dL}$ ). A plain radiograph of the abdomen (KUB) showed increased soft-tissue density and air-bubbles in the left inguinal region with ileus of the bowel loops (Fig. 1A). An ultrasound examination of the left inguinal region revealed a large heteroge- 
neously echogenic mass in the left scrotal sac (Fig. 1B), but the extent and origin of the lesion could not be well identified. A CT scan showed a tumor-like mass of the sigmoid colon surrounded by multiple abscesses within the left scrotal sac (Fig. 1C, D). The reformatted coronal CT images clearly showed the tumor herniating into the left scrotal sac through the inguinal canal, causing dilatation of the descending colon (Fig. 1D). There was no current evidence of peritoneal contamination.
After adequate resuscitation, an immediate surgical intervention was performed.

An exploratory laparotomy revealed the herniated sigmoid colonic loop, which was incarcerated and included the perforated colon cancer as well as the surrounding abscess pockets within the scrotal sac. Accordingly, the sigmoid segment was resected with an end colostomy and closure of the distal stump (Hartmann procedure). A hernia repair was also performed. The histopathology results

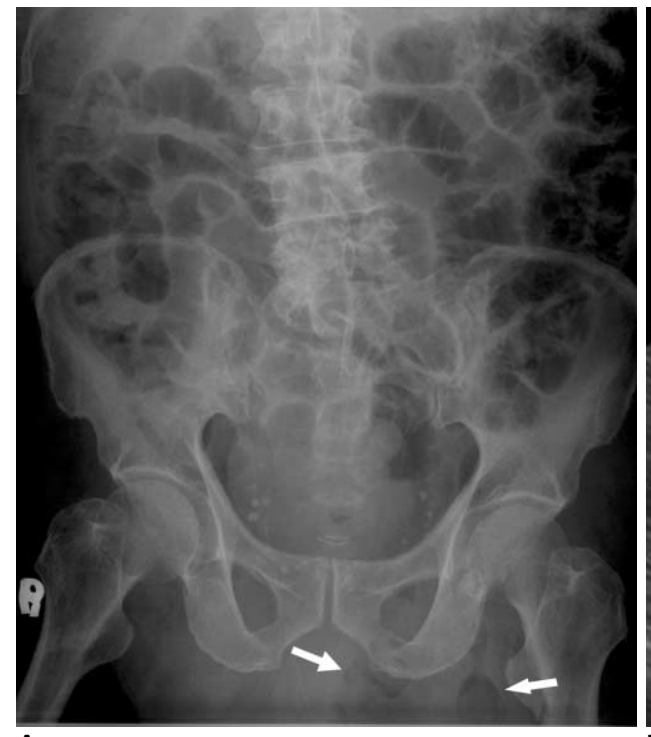

A

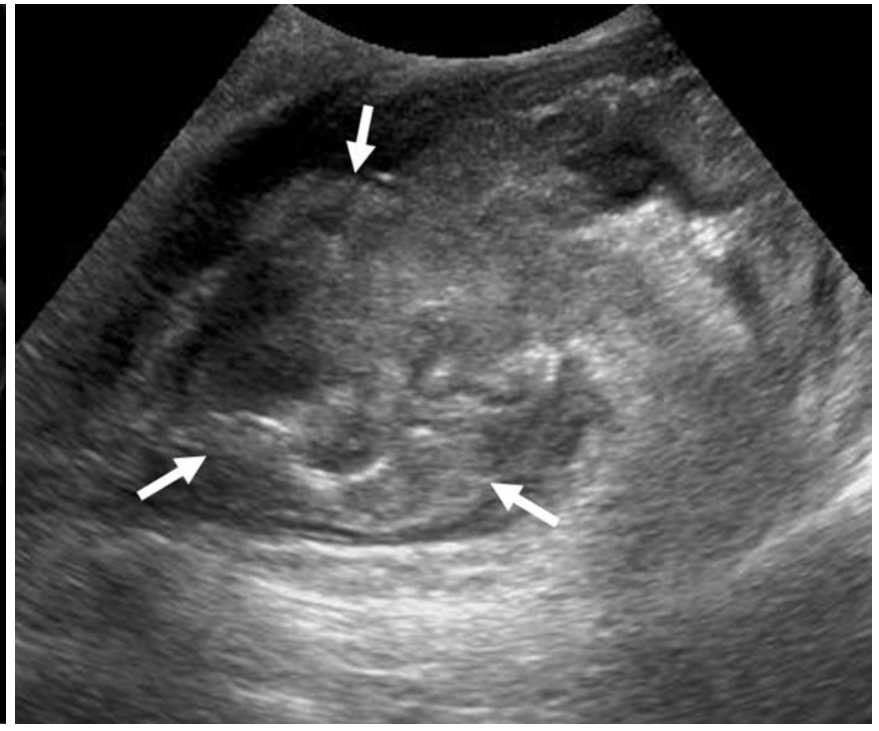

B

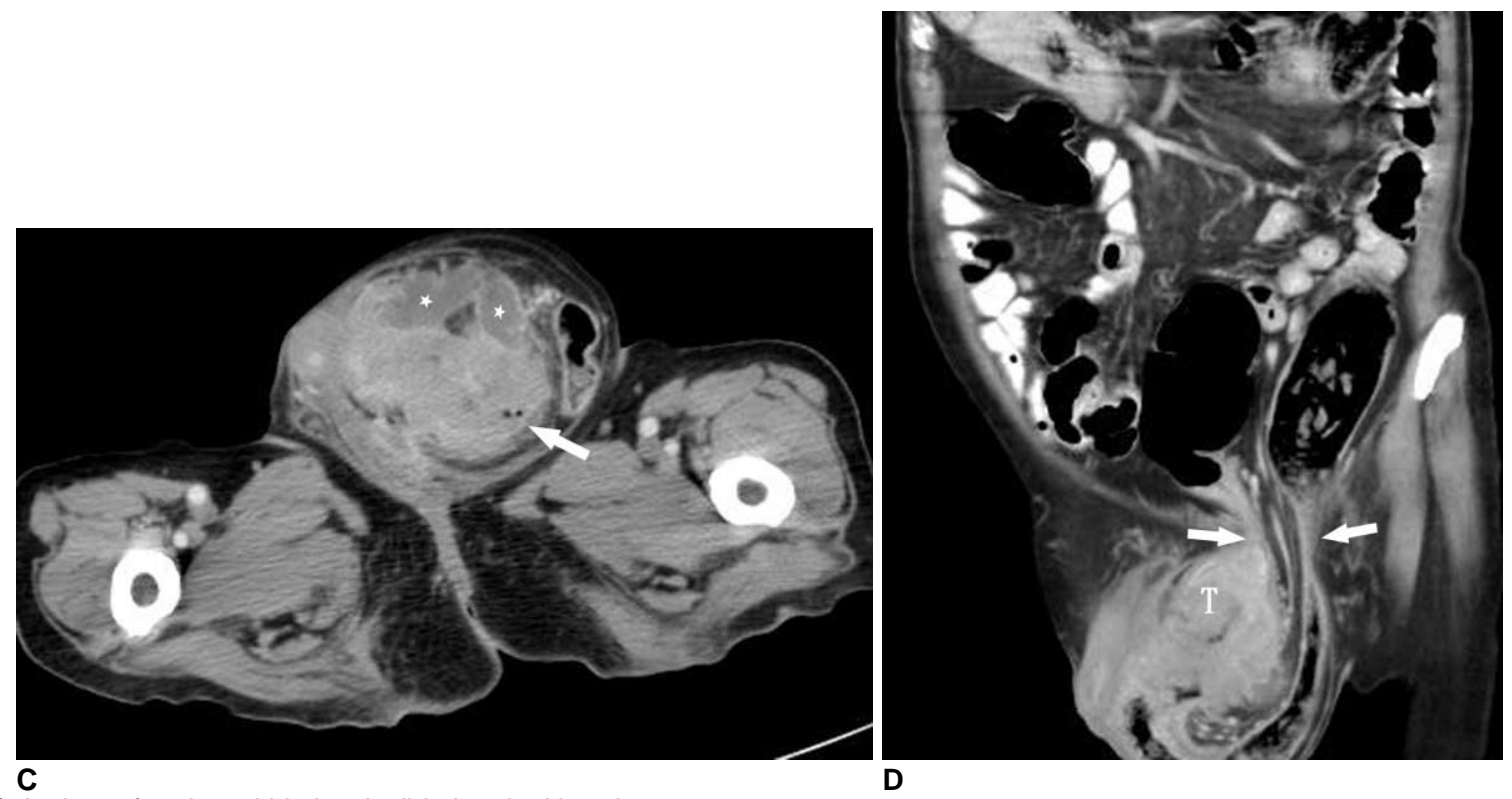

Fig. 1. Colonic perforation within irreducible inguinal hernia.

A. Plain abdominal radiograph shows increased soft tissue density including suspicious bowel-gas (arrows) in left inguinal region with ileus of intraabdominal bowel loops.

B. Ultrasound of scrotum shows large heterogeneously echogenic mass (arrows) in left scrotum with little fluid collection.

C. Axial CT image shows tumor-like mass of sigmoid colon (arrow) within left scrotal sac, which is surrounded by multiple abscesses (asterisks).

D. Reformatted coronal CT image shows sigmoid tumor (T) herniating into left scrotal sac through inguinal canal (arrows) and causing dilatation of descending colon. No evidence of peritoneal contamination is observed. 
confirmed the sigmoid adenocarcinoma diagnosis with moderate differentiation, tumor necrosis, as well as the invasion of all layers of the colon and pericolonic fatty tissue. Despite of the intensive postoperative care, acute respiratory failure by aspiration pneumonia was developed in the patient on the fifth postoperative day. Aggressive resuscitation efforts were not successful and the patient expired the next day due to septic shock.

\section{DISCUSSION}

An inguinal hernia is a common condition, in which, approximately $10 \%$ of cases reveal incarceration owing to benign or malignant entities (1). The prevalence of malignant tumors in inguinal hernias is rare - fewer than $0.4 \%$ of cases (2). These malignancies have been classified as saccular tumors (e.g. mesothelioma), metastatic disease involving the hernia sac itself, or intrasaccular tumors that refer to an organ bearing a primary malignancy within the hernia sac (3). Colon cancers are the most common malignancy of intrasaccular tumors and have been reported since 1938 (2). A review of the literature indicated that 27 cases have been described and that most of them were sigmoid adenocarcinomas in elderly patients, similar to our patient (1-6). However, having these comorbid conditions associated with perforation of the colon, as in our patient, is an extremely rare condition which has only been found in three reports.

As pointed out in previous studies, a perforated colorectal carcinoma is generally reported as having a poor prognosis because of high peri-operative mortality rate due associated with intra-abdominal sepsis and locally advanced malignancy (7). However, if the perforation in such a tumor is limited to the scrotal sac, without peritoneal contamination, there is a relatively good prognosis. On the other hand, any delay in diagnosis and management may lead to fatal complications such as necrotizing fasciitis involving the genitalia (8). In our patient, multiple abscesses around the sigmoid tumor were confined within the left scrotal sac and immediately identified by the CT scan. Although our patient had symptoms of sepsis and hyperglycemia that are predisposing factors for Fournier's gangrene, emergency surgery prevented it from progressing to this fatal sequela.

Traditionally, most inguinal hernia cases are confirmed by a physical examination, which then lead to surgery. An ultrasound may sometimes be helpful for the diagnosis. However, in complicated cases, such as in our particular case, an ultrasound and physical examination have a limited role in the detection of its etiology, extent, and surroundings. In a review of the previous 27 reported cases, most adenocarcinomas of the colon were found during surgery as an unexpected finding, and this may influence the surgical plan and post-operative treatment (1-6). In our patient, a pre-surgical CT clearly showed a perforated sigmoid tumor with abscess formation over the left inguinal region without peritoneal contamination or distant metastasis. Therefore, a CT should be suggested when an inguinal hernia becomes progressively enlarged, irreducible, and is accompanied by sepsis, anemia, weight loss, or bowel obstruction.

In conclusion, patients presenting with perforated sigmoid adenocarcinomas within inguinal hernias are rare, but this condition may have a catastrophic consequences. A CT is suggested as a rapid and reliable modality for facilitating a pre-operative diagnosis.

\section{References}

1. Boormans JL, Hesp WL, Teune TM, Plaisier PW. Carcinoma of the sigmoid presenting as a right inguinal hernia. Hernia 2006;10:93-96

2. Slater R, Amatya U, Shorthouse AJ. Colonic carcinoma presenting as strangulated inguinal hernia: report of two cases and review of the literature. Tech Coloproctol 2008;12:255-258

3. Tan GY, Guy RJ, Eu KW. Obstructing sigmoid cancer with local invasion in an incarcerated inguinal hernia. ANZ J Surg 2003;73:80-82

4. Kouraklis G, Kouskos E, Glinavou A, Raftopoulos J, Karatzas G. Perforated carcinoma of the sigmoid colon in an incarcerated inguinal hernia: report of a case. Surg Today 2003;33:707-708

5. Hale DA, Solla JA. Complete colonic obstruction caused by a sigmoid colon cancer incarcerated in an inguinal hernia sac. South Med J 1991;84:1280-1281

6. Sakorafas GH, Peros G. Obstructing sigmoid cancer in a patient with a large, tender, non-reducible inguinal hernia: the obvious diagnosis is not always the correct one. Eur J Cancer Care 2008;17:72-73

7. Mandava N, Kumar S, Pizzi WF, Aprile IJ. Perforated colorectal carcinomas. Am J Surg 1996;172:236-238

8. Baskin LS, Carroll PR, Cattolica EV, McAninch JW. Necrotising soft tissue infections of the perineum and genitalia. Bacteriology, treatment and risk assessment. Br J Urol 1990;65:524-529 